Doug Campos-Outcalt, MD, MPA

University of Arizona,

Phoenix

Ddougco@email.arizona edu

Dr. Campos-Outcalt is a member of the US Community Preventive Services Task Force.

doi: $10.12788 / \mathrm{jfp} .0318$

\title{
2021 CDC guidelines on sexually transmitted infections
}

\author{
A higher dose of ceftriaxone is now recommended for \\ gonorrhea. Doxycycline, not azithromycin, is first-line \\ therapy for chlamydia.
}

$\mathrm{I}$

July 2021, the Centers for Disease Control and Prevention (CDC) published its updated guidelines on the diagnosis, treatment, and prevention of sexually transmitted infections (STIs). ${ }^{1}$ These guidelines were last published in $2015 .^{2}$ Family physicians should be familiar with these guidelines as they are considered the standard of care for the treatment and prevention of STIs.

To revise the guidelines, the CDC convened a large panel that included CDC staff and subject matter experts from around the country. Using methodology borrowed from the US Preventive Services Task Force (USPSTF), ${ }^{3}$ the panel developed key questions and completed systematic reviews using a standard approach. The evidence behind key recommendations was ranked as high, medium, or low. However, the specific recommendations presented in the published guidelines appear without strength-of-recommendation descriptions or rankings of the levels of evidence supporting them.

The CDC approach to STI control involves 5 strategies (TABLE 1), ${ }^{1}$ which family physicians can implement as follows:

- Elicit an accurate sexual history.

- Discuss with patients and advise them on preventive interventions including barrier methods, microbicides, vaccines, and HIV pre-exposure prophylaxis.

- Order recommended screening tests for specific STIs from all sites of potential infection.
- Recognize the signs and symptoms of STIs and order recommended tests for confirmation.

- Treat confirmed infections using current recommended medications.

- Seek to advise, evaluate, and treat sex partners of those with documented STIs, and offer expedited partner therapy if allowed by state law.

- Perform recommended follow-up services for treated individuals.

Details on each of these strategies can be found in the new guidelines and are described for each specific pathogen and for specific demographic groups. Recommendations on screening for asymptomatic STIs can be found on the USPSTF website. ${ }^{4}$

The first step leading to targeted prevention strategies such as behavioral counseling, vaccination, and screening involves taking an accurate and complete sexual history. The CDC offers a 5-step process it calls the " 5 Ps approach" to gathering needed information (TABLE 2). ${ }^{1}$

\section{Major updates on the treatment of specific infections \\ Gonorrhea}

The current recommendation for treating uncomplicated gonococcal infections of the cervix, urethra, pharynx, and rectum in adults and adolescents weighing $<150 \mathrm{~kg}$ is ceftriaxone $500 \mathrm{mg}$ intramuscularly (IM) as a single 


\section{TABLE 1}

\section{Strategies to prevent and control STIs ${ }^{1}$}

1. Assess risk and educate and counsel at-risk individuals regarding ways to avoid STIs through changes in sexual behaviors and use of recommended prevention services.

2. Advise pre-exposure vaccination for vaccinepreventable STIs.

3. Identify individuals with an asymptomatic infection and those who have symptoms associated with an STI.

4. Diagnose, treat, counsel, and follow up with individuals infected with an STI.

5. Attempt to evaluate, treat, and counsel sex partners of individuals infected with an STI.

STIS, sexually transmitted infections.

dose; give $1 \mathrm{~g}$ for those weighing $\geq 150 \mathrm{~kg} .{ }^{1}$ If co-infection with chlamydia has not been ruled out, co-treatment with doxycycline $100 \mathrm{mg}$ po twice a day for 7 days is also recommended. ${ }^{1}$

This differs from the first-line treatment recommended in the previous guideline, which was dual therapy with ceftriaxone $250 \mathrm{mg}$ IM and azithromycin $1 \mathrm{~g}$ po as a single dose, regardless of testing results for chlamydia. ${ }^{2}$ The higher dose for ceftriaxone now recommended is due to a gradual decrease in gonorrhea susceptibility to cephalosporins in recent years, although complete resistance remains rare. The move away from universal dual therapy reflects a concern about antibiotic stewardship and the potential effects of antibiotics on the microbiome. The elimination of azithromycin from recommended firstline therapies is due to a 10 -fold increase in the proportion of bacterium isolates demonstrating reduced susceptibility, as measured by minimal inhibitory concentrations in the past few years.

If ceftriaxone is unavailable, there are 2 alternative regimens: gentamicin $240 \mathrm{mg}$ IM in a single dose, plus azithromycin $2 \mathrm{~g}$ po in a single dose; or cefixime $800 \mathrm{mg}$ po in a single dose. ${ }^{1}$ However, these alternatives are not recommended for gonococcal infection of the pharynx, for which ceftriaxone should be used.

Counsel those treated for gonorrhea to avoid sexual activity for 7 days after treatment
TABLE 2

\section{'5 Ps approach' for obtaining a sexual history ${ }^{1}$}

\section{Partners}

- "Are you currently having sex of any kind?"

- "What is the gender(s) of your partner(s)?"

\section{Practices}

- "To understand any risks for sexually transmitted infections (STIs), I need to ask more specific questions about the kind of sex you have had recently."

- "What kind of sexual contact do you have or have you had?"

- "Do you have vaginal sex, meaning 'penis in vagina' sex?"

- "Do you have anal sex, meaning 'penis in rectum/anus' sex?"

- "Do you have oral sex, meaning 'mouth on penis/vagina'?"

\section{Protection from STIs}

- "Do you and your partner(s) discuss prevention of STIs and human immunodeficiency virus (HIV)?"

- "Do you and your partner(s) discuss getting tested?"

- For condoms:

- "What protection methods do you use? In what situations do you use condoms?"

\section{Past history of STIs}

- "Have you ever been tested for STIs and HIV?"

- "Have you ever been diagnosed with an STI in the past?"

- "Have any of your partners had an STI?"

Additional questions for identifying HIV and viral hepatitis risk:

- "Have you or any of your partner(s) ever injected drugs?"

- "Is there anything about your sexual health that you have questions about?"

\section{Pregnancy intention}

- "Do you think you would like to have (more) children in the future?"

- "How important is it to you to prevent pregnancy (until then)?"

- "Are you or your partner using contraception or practicing any form of birth control?"

- "Would you like to talk about ways to prevent pregnancy?"

and until all sex partners have been treated. Because of the high rates of asymptomatic infections, tell patients to refer those with whom they have had sexual contact during the previous 60 days for evaluation, testing, and presumptive treatment.

Following treatment with the recommended dose of ceftriaxone, performing a test of cure is not recommended, with 1 exception: those with confirmed pharyngeal infection 


$\mid$\begin{tabular}{||l} 
TABLE 3 \\
Recommended parenteral \\
regimens for PID
\end{tabular}

cure is needed

following gonococcal

infection

treated with a

recommended

dose of

ceftriaxone, except in those with confirmed pharyngeal infection.

should be tested to confirm treatment success 7 to 14 days after being treated. However, all those treated for gonorrhea should be seen fection, regardless of whether they think their sex partners have been adequately treated.

\section{Chlamydia}

The recommended first-line therapy for chlamydia is now doxycycline $100 \mathrm{mg}$ twice a day be advised to seek evaluation, testing, and presumptive treatment.

\section{Trichomonas}

The recommended first-line treatment for trichomonas now differs for men and women: metronidazole $2 \mathrm{~g}$ po as a single dose for men, and metronidazole $500 \mathrm{mg}$ po twice a day for 7 days for women. ${ }^{1}$ Tinidazole $2 \mathrm{~g}$ po as a single dose is an alternative for both men and women. Previously, the single metronidazole dose was recommended for men and women, ${ }^{2}$ but there is now evidence that the 7-day course is markedly superior in achieving a cure in women.

No test of cure is recommended, but women should be retested at 3 months because of a high rate of re-infection. Current sex partners should be treated presumptively, and treated patients and their partners should avoid sex until all current sex partners have been treated. Consider expedited partner therapy if allowed by state law. again in 3 months and retested to rule out reinfor 7 days, which has proven to be superior to azithromycin (which was recommended as first-line therapy in 2015) for urogenital chlamydia in men and anal chlamydia in both men and women., Alternatives to doxycycline include azithromycin $1 \mathrm{~g}$ po as a single dose or levofloxacin $500 \mathrm{mg}$ po once a day for 7 days. ${ }^{1}$ No test of cure is recommended; but as with gonorrhea, retesting at 3 months is recommended because of the risk for reinfection.

Instruct patients treated for chlamydia to avoid sexual intercourse for 7 days after therapy is initiated or until symptoms, if present, have resolved. To reduce the chances of reinfection, advise treated individuals to abstain from sexual intercourse until all of their sex partners have been treated.

Sex partners in the 60 days prior to the patient's onset of symptoms or diagnosis should

\section{Bacterial vaginosis}

First-line treatment recommendations for bacterial vaginosis (BV) have not changed: metronidazole $500 \mathrm{mg}$ po twice a day for 7 days, or metronidazole gel $0.75 \%$ intravaginally daily for 5 days, or clindamycin cream $2 \%$ intravaginally at bedtime for 7 days. Advise women to avoid sexual activity or to use condoms for the duration of the treatment regimen.

A test of cure is not recommended if symptoms resolve, and no treatment or evaluation of sex partners is recommended. The guidelines describe several treatment options for women who have frequent, recurrent BV. To help prevent recurrences, they additionally suggest treating male partners with metronidazole $400 \mathrm{mg}$ po twice a day and with $2 \%$ clindamycin cream applied to the penis twice a day, both for 7 days.

\section{Pelvic inflammatory disease}

Recommended regimens for treating pelvic inflammatory disease (PID) have changed (TABLES 3 and 4). ${ }^{1}$ Women with mild or moderate PID can be treated with intramuscular or oral regimens, as outcomes with these regimens are equivalent to those seen with in- 
TABLE 4

\section{Recommended intramuscular or oral regimens for PID $^{1}$}

\footnotetext{
Ceftriaxone $500 \mathrm{mg}^{\mathrm{a}}$ IM in a single dose

plus

Doxycycline $100 \mathrm{mg}$ po bid for $14 \mathrm{~d}$, with metronidazole $500 \mathrm{mg}$ po bid for $14 \mathrm{~d}$

or

Cefoxitin $2 \mathrm{~g}$ IM in a single dose and probenecid $1 \mathrm{~g}$ po administered concurrently in a single dose

plus

Doxycycline $100 \mathrm{mg}$ po bid for $14 \mathrm{~d}$, with metronidazole $500 \mathrm{mg}$ po bid for $14 \mathrm{~d}$

or

Other parenteral third-generation cephalosporin (eg, ceftizoxime or cefotaxime)

plus

Doxycycline $100 \mathrm{mg}$ po bid for $14 \mathrm{~d}$ with metronidazole $500 \mathrm{mg}$ po bid for $14 \mathrm{~d}$

PID, pelvic inflammatory disease.

a For individuals weighing $\geq 150 \mathrm{~kg}$, administer ceftriaxone $1 \mathrm{~g}$.

travenous treatments. The nonintravenous options all include 3 antibiotics: a cephalosporin, doxycycline, and metronidazole.

To minimize disease transmission, instruct women to avoid sex until therapy is complete, their symptoms have resolved, and sex partners have been treated. Sex partners of those with PID in the 60 days prior to the onset of symptoms should be evaluated, tested, and presumptively treated for chlamydia and gonorrhea.
}

\section{Follow through on public health procedures}

STIs are an important set of diseases from a public health perspective. Family physicians have the opportunity to assist with the prevention and control of these infections through screening, making accurate diagnoses, and applying recommended treatments. When you suspect that a patient has an STI, test for the most common ones: gonorrhea, chlamydia, HIV, and syphilis. Report all confirmed diagnoses to the local public health department and be prepared to refer patients' sexual contacts to the local public health department or to provide contact evaluation and treatment.

Vaccines against STIs include hepatitis B vaccine, human papillomavirus vaccine, and hepatitis A vaccine. Offer these vaccines to all previously unvaccinated adolescents and young adults as per recommendations from the Advisory Committee on Immunization Practices. ${ }^{5}$

JFP

\section{References}

1. Workowski KA, Bachmann LH, Chan PA, et al. Sexually transmitted infections treatment guidelines, 2021. MMWR Recomm Rep. 2021;70:1-187.

2. Workowski KA, Bolan GA. Sexually transmitted diseases treat ment guidelines, 2015. MMWR Recomm Rep. 2015;64:1-137.

3. USPSTF. Methods and processes. Accessed November 17 2021. https://uspreventiveservicestaskforce.org/uspstf/aboutuspstf/methods-and-processes

4. USPSTF. Recommendations. Infectious diseases. Accessed November 17, 2021. https://uspreventiveservicestaskforce.org/ uspstf/topic_search_results?topic_status=P\&category\%5B\%5D $=18 \&$ searchterm $=$

5. CDC. Advisory Committee on Immunization Practices. COVID-19 ACIP vaccine recommendations. Accessed October 18, 2021. www.cdc.gov/vaccines/hcp/acip-recs/vacc-specific/ covid-19.html
CONTINUED FROM PAGE 505

positive women in cervical cancer screening. J Clin Virol 2016;76 S49-S55. doi: 10.1016/j.jcv.2015.11.015

23. WHO Guidelines: Use of Cryotherapy for Cervical Intraepithe lial Neoplasia. Geneva, Switzerland: World Health Organization 2011. Accessed November 14, 2021. www.ncbi.nlm.nih.gov/ books/NBK138476/pdf/Bookshelf_NBK138476.pdf

24. Spence AR, Goggin P, Franco EL. Process of care failures in invasive cervical cancer: systematic review and meta-analysis. Prev Med. 2007:45:93-106. doi: 10.1016/j.ypmed.2007.06.007

25. Rositch AF, Nowak RG, Gravitt PE. Increased age and race-specific incidence of cervical cancer after correction for hysterectomy prevalence in the United States from 2000-2009. Cancer. 2014:120:2032-2038. doi: 10.1002/cncr.28548

26. Siegel RL, Miller KD, Fuchs HE, et al. Cancer statistics, 2021. CA Cancer J Clin. 2021;71:7-33. doi: 10.3322/caac.21654

27. National Comprehensive Cancer Network. Clinical practice guidelines in oncology: cervical cancer. Accessed June 15, 2021 www.nccn.org/professionals/physician_gls/pdf/cervical.pdf
28. Tewari KS, Sill MW, Penson RT, et al. Bevacizumab for advanced cervical cancer: final overall survival and adverse event analysis of a randomised, controlled, open-label, phase 3 trial (Gynecologic Oncology Group 240). Lancet. 2017;390:1654-1663. doi: 10.1016/S0140-6736(17)31607-0

29. Osann K, Hsieh S, Nelson EL, et al. Factors associated with poor quality of life among cervical cancer survivors: implications for clinical care and clinical trials. Gynecol Oncol. 2014;135:266272. doi: 10.1016/j.ygyno.2014.08.036

30. Ries LAG, Harkins D, Krapcho M, et al. SEER Cancer Statistic Review, 1975 to 2003. Bethesda, MD: National Cancer Institute 2007. Accessed November 14, 2021. https://seer.cancer.gov/ archive/csr/1975_2003/\#citation

31. Hu Z, Ding M. The precision prevention and therapy of HPV related cervical cancer: new concepts and clinical implications Cancer Med. 2018;7:5217-5236. doi: 10.1002/cam4.1501

32. Wang R, Pan W, Jin L, et al. Human papillomavirus vaccine against cervical cancer: opportunity and challenge. Cancer Lett. 2020;471:88-102. doi: 10.1016/j.canlet.2019.11.039 